\title{
Naming cAsE aLtErNaTeD words
}

\author{
CHRIS M. HERDMAN and DONNA CHERNECKI \\ Carleton University, Ottawa, Ontario, Canada \\ and \\ DENNIS NORRIS \\ MRC Applied Psychology Unit, Cambridge, England
}

\begin{abstract}
A dual-route approach was used as an initial framework to examine the relation between presentation format and lexical processing in a naming task. In Experiments 1 and 3, words were presented in lowercase versus case-alternated format. Presentation format interacted with word frequency and regularity: For irregular words (e.g., pint), case alternation was additive with frequency, whereas for regular words (e.g., mint), case alternation and frequency interacted. Experiment 2 dissociated the locus of case-alternation effects from those of stimulus intensity. Stimulus intensity was additive with frequency and regularity, suggesting that whereas stimulus intensity affects encoding, case alternation affects lexical processing at a postencoding stage in the word recognition system. It was concluded that a dualroute approach provides a suggestive but incomplete account of how case alternation influences lexical processing. As an alternative to a dual-route approach, we show that the present results can be addressed and successfully simulated using an implemented version of Norris's (1994) multilevel model.
\end{abstract}

Theorists in the word recognition literature have centered their models on the assumption that variability in lexical processing can largely be accounted for by two classes of variables: orthographic factors,.such as the regularity of a word's spelling-to-sound correspondences, and experiential factors, such as the frequency of a word's occurrence in printed text (e.g., Coltheart, Curtis, Atkins, \& Haller, 1993; Morton, 1969; Norris, 1994; Paap, McDonald, Schvaneveldt, \& Noel, 1987; Paap \& Noel, 1991; Seidenberg \& McClelland, 1989). There is evidence, however, that lexical processing is also influenced by variations in presentation format that disrupt the interletter relations in a word (Besner, 1990; Besner \& Johnston, 1989; Besner \& McCann, 1987; see also Adams, 1979; Coltheart \& Freeman, 1974; Masson, 1986). For example, Besner and McCann (1987) required subjects to name high- and low-frequency words presented in either lowercase or case-alternated format (e.g., aLtErNaTiOn). The majority of the words in their experiment were regular in that they followed consistent spelling-to-sound patterns (e.g., mint). An interaction between case alternation and fre-

This research was supported by the Natural Sciences and Engineering Research Council of Canada (NSERC) through grants to C.M.H. and by an NSERC postgraduate scholarship to D.C. C.M.H. is grateful to the MRC Applied Psychology Unit in Cambridge, England, for providing a supportive environment during the writing of this article. We thank Geoff Loftus, Colin Macleod, Mike Masson, Ken Paap, and an anonymous reviewer for insightful critiques of earlier versions of this article. This research also benefited immensely from many stimulating conversations with Jo-Anne LeFevre. Correspondence should be addressed to C. M. Herdman, Department of Psychology, Carleton University, Ottawa, ON K1S 5B6, Canada (e-mail: cherdman@ccs.carleton.ca).

—Accepted for publication by previous editor, Geoffrey $R$. Loftus quency was found such that case alternation slowed naming more to low-frequency than to high-frequency words. In a subsequent study, Besner (1990) found that when subjects were presented with lists containing only irregular words (e.g., pint), case alternation and frequency were additive: Case alternation slowed naming of high- and lowfrequency irregular words equally. Insofar as interactions between case alternation and frequency or case alternation and regularity implicate processing within a common stage in the word recognition system, the findings of Besner and colleagues indicate that theorists need to extend their models to account for the influence of presentation format on lexical processing.

In the present research, we investigated the influence of presentation format on lexical processing by manipulating case alternation, frequency, and regularity in a naming task. Following Besner and colleagues (Besner, 1990; Besner \& Johnston, 1989; Besner \& McCann, 1987), we used a dual-route approach as the initial framework for this research. The results of the present research, however, showed that a dual-route approach provides an incomplete account of how case alternation influences the word recognition process. As an alternative to a dual-route framework, we interpreted the effects of case alternation using Norris's (1994) multilevel model. An implemented version of the multilevel model is described in the General Discussion and is shown to successfully simulate the results of the present research.

\section{Dual-Route Framework}

Figure 1 shows a version of the dual-route architecture used by Besner and colleagues to describe how case alternation influences processing in the word recognition system. ${ }^{1}$ As shown in Figure 1, processing initially involves 


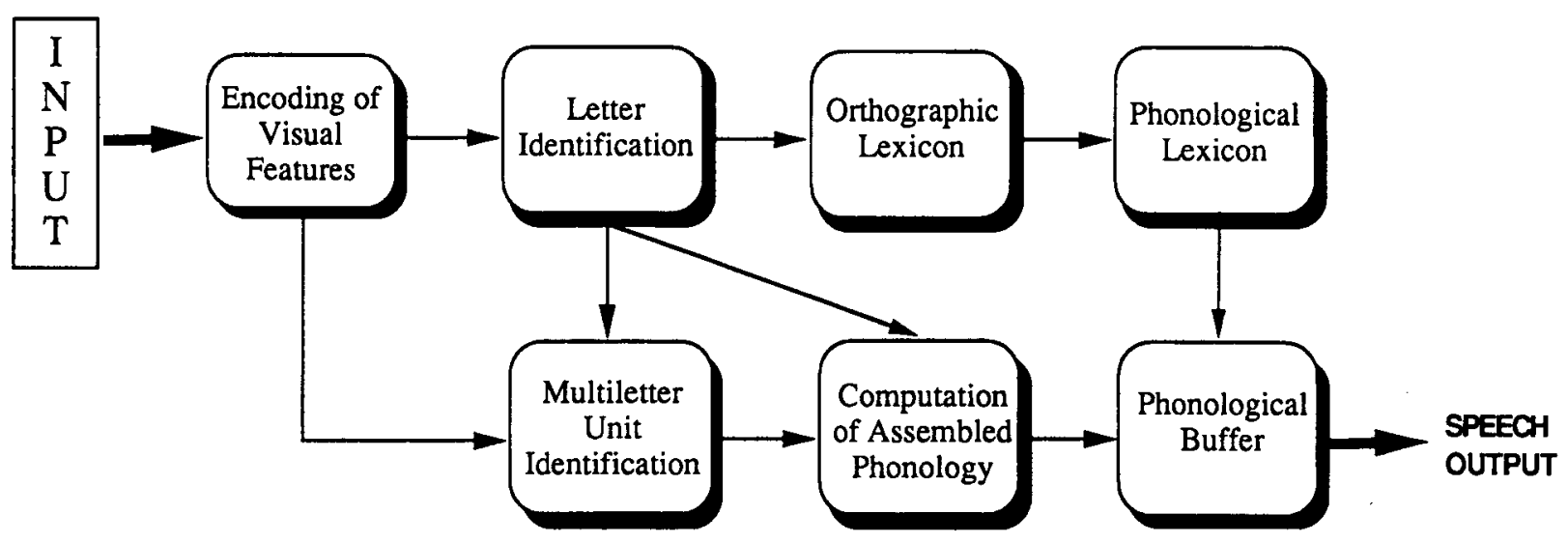

Figure 1. Dual-route model adapted from Besner and Johnston (1989).

preliminary letter and multiletter identification stages that feed into a lexical and a nonlexical route. For the lexical route, information is fed from the letter identification stage into an orthographic lexicon, which in turn is directly connected to a phonological lexicon. Representations in the lexicons or the links connecting the lexicons (McCann \& Besner, 1987) are assumed to be sensitive to frequency in such a way that high-frequency words are processed more rapidly than low-frequency words. For the nonlexical route, information from the preliminary letter and multiletter stages is converted into corresponding phonemes, which are then assembled into larger phonolegical units. Because the nonlexical route operates at a sublexical level, it is presumably insensitive to word frequency. Both routes feed codes into a phonological buffer that contains decision/synthesis mechanisms to resolve conflicts between the lexical and nonlexical codes and to synthesize a phonology for output (Carr \& Pollatsek, 1985; see also Herdman \& Beckett, 1996; Herdman, LeFevre, \& Greenham, 1994; Monsell, Patterson, Graham, Hughes, \& Milroy, 1992).

Besner and colleagues have proposed two separate accounts of how case alternation influences processing in a dual-route system. We refer to this as the two-account/ dual-route approach. Account 1 addresses the results of Besner and McCann (1987), in which case alternation and frequency were found to interact. Account 2 addresses the results of Besner (1990), in which case alternation and frequency were additive.

Account 1: Differential slowing of the routes. Account 1 was designed to address Besner and McCann's (1987) finding that case alternation slowed naming more to low-than to high-frequency regular words. To address this finding, Besner and Johnston (1989) suggested that case alternation slows processing along both the lexical and the nonlexical route at the letter identification stage: A larger set of letters must be searched when stimuli are presented in case-alternated as opposed to lowercase format (potentially 52 vs. 26 letters, respectively). In addition, case alternation is assumed to slow nonlexical process- ing at the multiletter identification stage: The identification of adjacent letters that correspond to phonemes is impaired because letter combinations form uncommon visual patterns (e.g., $c H$ rather than $c h$ ). According to Besner and Johnston, therefore, case alternation slows processing throughout the word recognition system, but more so along the nonlexical than the lexical route.

In a dual-route framework, high-frequency words are processed rapidly via the frequency-sensitive lexical route and with little or no recourse to the nonlexical route. Accordingly, case alternation should slow naming of highfrequency words only via disruption of the preliminary letter identification stage. Phonological coding of lowfrequency words, on the other hand, often relies on output from both the lexical and the nonlexical routes. If both of these routes are impaired by case alternation, naming of low-frequency words should be slowed substantially. In sum, the assumption that case alternation slows processing more along the nonlexical than the lexical route can be used to explain the Besner and McCann's (1987) finding that case alternation slowed naming more to lowthan to high-frequency words. ${ }^{2}$

Account 2: Strategic orienting toward the lexical route. Account 2 was specifically formulated to address Besner's (1990) finding that case alternation slowed naming of high- and low-frequency words equally when subjects were presented with lists containing only irregular words (e.g., pint). To address this finding, Besner suggested that presenting lists containing irregular words strategically orients processing away from the nonlexical route and toward the lexical route (for orienting effects, see also Baluch \& Besner, 1991; Herdman et al., 1994; Monsell et al., 1992; Peereman \& Content, 1995; Pugh, Rexer, \& Katz, 1994). This list-induced orienting presumably circumvents processing along the nonlexical route and thereby eliminates the additional source of slowing associated with processing low-frequency words in casealternated format (see Account 1). Because the effects of case alternation were undifferentiated across high- versus low-frequency irregular words, Besner assumed that 
case alternation influences the letter identification stage and that this stage is discrete from frequency-sensitive processes in the orthographic lexicon (see note 2).

\section{Present Research}

Besner and colleagues' two accounts of how case alternation affects processing in the word recognition system can coexist within a dual-route framework: The assumption that processing can be strategically oriented toward the lexical route (Account 2) does not compromise the assumption that case alternation slows processing more along the nonlexical route than the lexical route (Account 1), or vice versa. Importantly, however, although these two accounts may coexist, there are situations where they cannot be simultaneously applied.

Consider a situation in which subjects are presented with a mixed list for which half of the items are regular words and half irregular words. If the presence of the irregular words in the list is sufficient to orient processing toward the lexical route, then Account 2 would apply, in which slowing of processing along the lexical route is assumed to be undifferentiated across frequency. Accordingly, case alternation and frequency should be additive, and this additivity should be found for both irregular words (as in Besner, 1990) and regular words. However, if the presence of the irregular words in the list does not cause processing to be oriented toward the lexical route, then Account 1 would apply, and it must be assumed that case alternation slows processing more along the nonlexical than the lexical route. For regular words, the interaction reported by Besner and McCann (1987) would be predicted, where case alternation slowed naming more to low- than to high-frequency words. Similarly, a case alternation $X$ frequency interaction would also be predicted for irregular words. Interestingly, however, the nature of this interaction would differ from that for regular words. Because the nonlexical route generates incorrect phonologies to low-frequency irregular words, extra slowing of this route relative to the lexical route should actually benefit naming of these words: The (incorrect) nonlexical code would be less likely to interfere with the (correct) lexical code in the phonological buffer (see Bernstein \& Carr, 1996; Herdman \& Beckett, 1996; Paap \& Noel, 1991), thereby facilitating naming performance. It should also be noted that, on the basis of Account 1, case alternation should have equivalent effects on the naming of both high-frequency regular and high-frequency irregular words: In a dual-route framework, all highfrequency words are processed via the lexical route, so additional slowing of the nonlexical route should not affect these words.

In sum, the two-account/dual-route approach hinges on applying either Account 1 or Account 2 to a particular data set. However, the critical test of the two-account approach has not been conducted: The relationship between case alternation and frequency has not been examined under mixed-list conditions in which both regular and irregular words are presented. To this end, in Experiments
1 and 3 of the present research, case alternation, frequency, and regularity were combined into a single experimental design. Experiment 2 was conducted as a control study to dissociate the locus of case-alternation effects from those of stimulus intensity.

\section{EXPERIMENT 1}

In Experiment 1, subjects were required to name words presented either in lowercase or in case-alternated format: Word frequency and regularity were manipulated factorially. Regular and irregular words were presented in a mixed list, thereby providing a critical test of the two-account/dual-route framework. Because the two accounts cannot be applied simultaneously, one set of predictions was made on the basis of Account 1 and another set was made on the basis of Account 2.

If the presence of irregular words in the mixed list did not orient processing toward the lexical route, then Account 1 (differential slowing of the routes) would apply and case alternation should interact with frequency. For regular words, the pattern reported by Besner and McCann (1987) should be found in which case alternation slowed naming more to low- than to high-frequency words. For irregular words, a case alternation $\times$ frequency interaction should be found; however, this interaction should differ from that for regular words. Of particular note, and as discussed above, naming of low-frequency irregular words should be faster in the case-alternated than in the lowercase condition because of the reduced conflict from incorrect phonologies produced along the nonlexical route.

In contrast, if the presence of irregular words in the mixed list caused processing to be oriented toward the lexical route, then Account 2 would apply. On this view, the effects of case alternation should solely reflect slower processing along the lexical route via disruption at the letter identification stage. Insofar as the letter identification stage is discrete from processing in the orthographic lexicon (Besner, 1990; see also note 2), this slowing should be undifferentiated across frequency and regularity. In sum, the orienting account predicts additive effects of case alternation.

\section{Method}

Subjects. A total of 40 undergraduate students at Carleton University ( 20 male, 20 female) were recruited to participate. They received either course credit or $\$ 5$. All of the subjects had normal or corrected-to-normal vision and were native English speakers.

Apparatus. An IBM-type 80286 computer equipped with a Digitek Electronics input-output (I/O) board was used to present letter strings on a Samsung SF12 video screen. Luminance was set at 17.2 fLs. ${ }^{3}$ Naming responses were detected using a Sharpe LM6 boom microphone mounted to a set of Scintrex headphones and connected to a Grason-Stadler Model E7300A-I voice-activated relay, which was interfaced to the Digitek I/O board. Subjects were seated approximately $50 \mathrm{~cm}$ from the video monitor. A microswitch located on a button box was used to initiate trials. Response latencies were accurate to $1 \mathrm{msec}$.

Design and Materials. A 2 (case: lower vs, alternated) $\times 2$ (frequency: high vs. low) $\times 2$ (regularity: regular vs. irregular) re- 
peated measures design was used. Four categories of words were created by crossing frequency and regularity. There were 20 monosyllabic words in each of the four categories for a total of 80 words (see the Appendix). The words were selected in such a way that (1) there were equal numbers of high- and low-frequency words in the categories, (2) frequency was balanced across regularity, (3) the words in each frequency $\times$ regularity group were matched for length, and (4) with the exception of the low-frequency regular words plank and sank, no spelling patterns were repeated in the categories. Classification of words as irregular was based on criteria used by Waters and Seidenberg (1985). Only irregular words that have regular spelling patterns but irregular pronunciations were chosen (words with uncommon spellings such as yacht and ache were not included). Four stimulus orders were used and each subject saw one order. In each order, half of the words were case-alternated and half were in lowercase. Case was balanced across order in such a way that each word appeared in lowercase in two of the four orders and in case-alternated format in the other two orders. Each order was constructed randomly with the restrictions that no more than three lowercase or case-alternated words appeared consecutively and that frequency and regularity were represented equally in each half of each order.

Procedure. Subjects were tested individually in sessions lasting approximately $30 \mathrm{~min}$. A trial began with the presentation of an asterisk $\left({ }^{*}\right)$ in the center of the video screen. Subjects fixated on the asterisk and then pushed a microswitch to initiate a trial. Upon initiation of a trial, the asterisk disappeared and was replaced $300 \mathrm{msec}$ later by a word. The word was written either in lowercase (e.g., give) or in alternated case (e.g., gIvE), with the first letter always in lowercase. The word remained on the screen until it was pronounced. A variable intertrial interval elapsed while the experimenter recorded whether the pronunciation of the word was correct. The 80 experimental trials were preceded by 16 practice trials.
Subjects were instructed to pronounce the words as quickly and as accurately as possible.

\section{Results and Discussion}

Latencies. Latencies to correct responses are shown in Figure 2 (left panel) and in Table 1. Incorrect responses and trials on which the equipment failed were not included in the latency data. Latencies were examined with a 2 (case: lower vs. alternated) $\times 2$ (frequency: high vs. low) $\times 2$ (regularity: regular vs. irregular) repeated measures analysis of variance (ANOVA). The ANOVA summary is shown in Table 2. All three main effects were significant. Words presented in lowercase were named faster than words presented in alternated case, high-frequency words were named faster than low-frequency words, and regular words were named faster than irregular words.

Research has shown that frequency and regularity interact in naming tasks (Seidenberg, Waters, Barnes, \& Tannenhaus, 1984). Similarly, in the present experiment, a significant frequency $\times$ regularity interaction showed that latencies did not differ substantially between highfrequency regular and irregular words, whereas latencies were faster to low-frequency regular than irregular words. The interaction between case and regularity was also significant. In the lowercase condition, there was a small difference in naming latencies for irregular versus regular words. In the alternated-case condition, however, latencies were substantially greater for irregular than for regular words.

\section{Experiment 1 Experiment 2 Experiment 3}

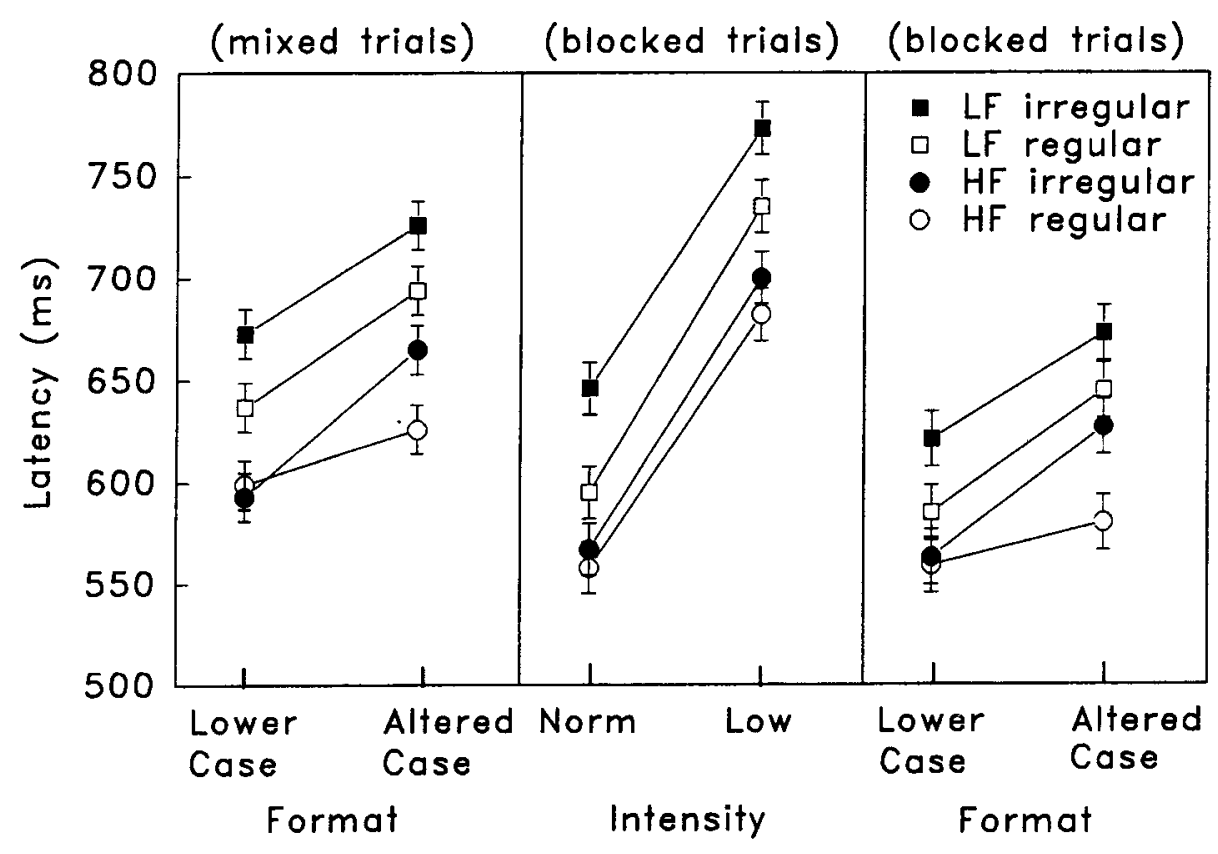

Figure 2. Naming latencies in Experiments 1-3. Bars are $95 \%$ confidence intervals calculated using the $M S_{\mathrm{e}}$ corresponding to the three-way effects for each experiment. 
Table 1

Reaction Times (RTs, in Milliseconds), Standard Deviations, and Percent Naming Errors (PE)

\begin{tabular}{|c|c|c|c|c|c|c|c|c|c|c|c|}
\hline \multirow[b]{2}{*}{ Frequency } & \multicolumn{3}{|c|}{ Regular } & \multicolumn{2}{|c|}{ Irregular } & \multicolumn{3}{|c|}{ Regular } & \multicolumn{3}{|c|}{ Irregular } \\
\hline & RT & $S D$ & $\mathrm{PE}$ & RT & $\overline{P E}$ & RT & $S D$ & $\mathrm{PE}$ & RT & $S D$ & $P E$ \\
\hline & \multicolumn{11}{|c|}{ Experiment 1} \\
\hline & \multicolumn{5}{|c|}{ Lowercase } & \multicolumn{6}{|c|}{ Case Alternated } \\
\hline High & 599 & 83.4 & 1.8 & 593 & $90.6 \quad 2.5$ & 626 & 106.3 & 3.5 & 665 & 117.0 & 3.8 \\
\hline \multirow[t]{3}{*}{ Low } & 637 & 115.9 & 5.3 & 673 & $120.1 \quad 18.0$ & 694 & 133.9 & 8.5 & 726 & 144.2 & 18.3 \\
\hline & \multicolumn{11}{|c|}{ Experiment 2} \\
\hline & \multicolumn{5}{|c|}{ Normal Intensity } & \multicolumn{6}{|c|}{ Low Intensity } \\
\hline High & 558 & 61.5 & 2.5 & 568 & $\begin{array}{ll}71.7 & 0.8\end{array}$ & 682 & 99.6 & 2.0 & 700 & 104.9 & 3.3 \\
\hline \multirow[t]{3}{*}{ Low } & 595 & 74.4 & 3.8 & 646 & $127.4 \quad 15.8$ & 735 & 129.3 & 3.5 & 773 & 147.5 & 16.5 \\
\hline & \multicolumn{11}{|c|}{ Experiment 3} \\
\hline & \multicolumn{5}{|c|}{ Lowercase } & \multicolumn{6}{|c|}{ Case Alternated } \\
\hline High & 559 & 77.1 & 0.3 & 562 & $77.0 \quad 0.8$ & 580 & 67.6 & 1.0 & 627 & 98.4 & 3.3 \\
\hline Low & 585 & 77.5 & 4.3 & 621 & $91.3 \quad 13.3$ & 645 & 107.6 & 6.8 & 673 & 123.3 & 16.3 \\
\hline
\end{tabular}

Of primary interest was the significant three-way interaction of case $\times$ frequency $\times$ regularity. As shown in Figure 2 (left panel), the pattern of results replicates both that of Besner and McCann (1987) and that of Besner (1990). For regular words, case alternation slowed naming more to low- than to high-frequency words. For irregular words, case alternation slowed naming of lowand high-frequency words equally. These patterns were confirmed with separate 2 (case: lower vs. alternated) $\times$ 2 (frequency: high vs. low) analyses (Table 2). For regu lar words, there were significant main effects of case and frequency and, more importantly, a significant case $\times$ frequency interaction that replicates the pattern of results reported by Besner and McCann. For irregular words, there were main effects of case and frequency. However, these two factors did not interact, thus replicating Besner's evidence of additivity between case alternation and frequency for irregular words.

Errors. Percent naming errors (see Table 1) were analyzed with a 2 (case: lower vs. alternated) $\times 2$ (frequency: high vs. low) $\times 2$ (regularity: regular vs. irregular) repeated measures ANOVA (see summary in Table 2). More errors were made pronouncing low-frequency $(12.5 \%)$ than high-frequency $(2.9 \%)$ words and irregular $(10.6 \%)$ than regular $(4.8 \%)$ words. The frequency $\times$ regularity interaction found with latencies was supported in the error data: For high-frequency words, there was a small difference in errors to irregular $(3.1 \%)$ versus regular words $(2.6 \%)$. For low-frequency words, there were substantially more errors to irregular (18.1\%) than to regular words $(6.9 \%)$.

Summary. The results of Experiment 1 show that for regular words, there was an interaction between case alternation and frequency, whereas for irregular words, case alternation and frequency were additive. According to the two-account/dual-route framework, these two patterns of results should not have occurred concomitantly.

Consider the possibility that the presence of irregular words in the stimulus list did not cause processing to be strategically oriented toward the lexical route. The interaction between case alternation and frequency that was found with regular words could be explained using Account 1 , in which it is assumed that case alternation slows the nonlexical route more than the lexical route (Besner \& Johnston, 1989). On the basis of this account, however, case alternation should not have slowed naming more to high-frequency irregular than to high-frequency regular words. ${ }^{4}$ In a dual-route framework, slowing of processing along the nonlexical route should not affect high-frequency words because all high-frequency words are named solely on the basis of output from the lexical route. Moreover, the Account 1 assumption that processing along the non-

Table 2

ANOVA Summaries for the Overall and the By-Regularity Analyses in Experiment 1

\begin{tabular}{lccc}
\hline \multicolumn{1}{c}{ Source } & $M S_{\mathrm{e}}$ & $F$ & $p$ \\
\hline Latencies: Overall analysis & & & \\
$\quad$ Case & 3,828 & 57.03 & $<.001$ \\
Frequency & 3,641 & 83.39 & $<.001$ \\
Regularity & 2,173 & 24.16 & $<.001$ \\
Case $\times$ frequency & 740 & $<1$ & n.s. \\
Case $\times$ regularity & 1,954 & 4.18 & $<.049$ \\
Frequency $\times$ regularity & 1,130 & 5.08 & $<.031$ \\
Case $\times$ frequency $\times$ regularity & 1,385 & 8.49 & $<.007$ \\
Latencies: By-regularity analyses & & & \\
Regular words & & & \\
$\quad$ Case & 1,625 & 43.68 & $<.001$ \\
$\quad$ Frequency & 2,337 & 48.32 & $<.001$ \\
$\quad$ Case $\times$ frequency & 1,615 & 5.70 & $<.023$ \\
Irregular words & & & \\
$\quad$ Case & 4,158 & 37.40 & $<.001$ \\
$\quad$ Frequency & 2,434 & 80.72 & $<.001$ \\
$\quad$ Case $\times$ frequency & 1,513 & 2.18 & n.s. \\
Errors: Overall analysis & & & \\
Case & 71.51 & 2.95 & n.s. \\
Frequency & 88.17 & 84.05 & $<.001$ \\
Regularity & 85.61 & 35.25 & $<.001$ \\
Case $\times$ frequency & 61.51 & $<1$ & n.s. \\
Case $\times$ regularity & 74.07 & $<1$ & n.s. \\
Frequency $\times$ regularity & 66.38 & 34.82 & $<.001$ \\
Case $\times$ frequency $\times$ regularity & 54.33 & $<1$ & n.s. \\
\hline & & & \\
& & &
\end{tabular}


lexical route is especially slowed by case alternation does not explain why case alternation slowed naming of lowfrequency regular and irregular words equally (see Figure 2, left panel): As noted, low-frequency irregular words should have benefited from slowing of the nonlexical route because there would presumably be less interference in the buffer from the incorrect nonlexical code.

Next, consider Besner's (1990) second account, in which additivity between case alternation and frequency is assumed to reflect strategic orienting of processing toward the lexical route. On this view, additivity between case alternation and frequency should have occurred not only with irregular words but also with regular words. In fact, the orienting assumption erroneously predicts that case alternation should have resulted in slowing that was undifferentiated across frequency and regularity.

\section{EXPERIMENT 2}

The results of Experiment 1 show that case alternation interacts with lexical factors (frequency and regularity). This finding suggests that case alternation does not simply slow the rate at which information is encoded. Rather, case alternation appears to affect processing at a postencoding stage in the word recognition system. Experiment 2 was conducted to substantiate this postencoding interpretation of case-alternation effects by establishing that, for the set of words and lexical factors examined in the present research, changes in the rate at which words are encoded does indeed produce additive effects. To this end, stimulus intensity was manipulated by requiring subjects to name words presented in either normal or low-illumination conditions. As in Experiment 1, word frequency and regularity were varied factorially.

Stimulus intensity has seldom been manipulated in naming tasks (but see Besner \& Smith, 1992), and to the best of our knowledge, the relation between stimulus intensity, frequency, and regularity has not been examined. In lexical decisions, additivity between stimulus intensity and frequency has been reported in several studies (e.g., Besner \& Swan, 1982; Paap et al., 1987; Stanners, Jastrzembski, \& Westbrook, 1975). In accord with additive factors logic (Sternberg, 1969), these findings suggest that stimulus intensity affects an encoding stage that exists prior to and independent of frequency-sensitive processes in the word recognition system. On this view, and in contrast to the interaction of case alternation, frequency, and regularity found in Experiment 1, we predicted that stimulus intensity would be additive with frequency and regularity in the present experiment.

\section{Method}

Subjects. A total of 40 undergraduate students at Carleton University ( 20 male, 20 female) were recruited to participate; they received either course credit or $\$ 5$. All of the subjects had normal or corrected-to-normal vision and were native English speakers. None of the subjects had participated in Experiment 1.

Apparatus, Design, and Materials. The apparatus and design were identical to those used in Experiment 1, with the exception that stimulus intensity (normal vs. low) replaced case alternation. The same stimulus set of 80 words was used. These words were separated into two blocks of 40 words. As in Experiment 1, four orders were used. Each order consisted of two blocks. Each block contained 20 high-and 20 low-frequency words, with half of each being regular and the other half irregular. For one block, items were presented under normal viewing conditions with luminance set at $17.2 \mathrm{fLs}$. For the low-intensity block, luminance was reduced to $0.69 \mathrm{fLs}$ by placing a filter consisting of three sheets of colored transparency film (two green and one red, selected on the basis of pilot research) over the video screen.

Procedure. The procedure was identical to that of Experiment 1 with the exception that blocked presentation was used. The order of blocks (i.e., normal vs. low illumination) was counterbalanced across subjects. Subjects completed 10 practice trials before each of the 40 normal illumination and 40 low-illumination blocks of trials.

\section{Results and Discussion}

Latencies. Latencies to correct responses are shown in Figure 2 (middle panel) and Table 1. Latencies were examined with a 2 (stimulus intensity: normal vs. low) $\times 2$ (frequency: high vs. low) $\times 2$ (regularity: regular vs. irregular) repeated measures ANOVA. The ANOVA summary is shown in Table 3. All three main effects were significant. Words were named faster in normal than in lowillumination viewing conditions, high-frequency words were named faster than low-frequency words, and regular words were named faster than irregular words.

The interaction between frequency and regularity reported in the literature (e.g., Seidenberg et al., 1984) and in Experiment 1 was replicated. For high-frequency words, there was a relatively small difference in latencies for regular versus irregular items. In contrast, for lowfrequency words, latencies were substantially faster to

Table 3

ANOVA Summaries for the Overall and the By-Regularity Analyses in Experiment 2

\begin{tabular}{lrcc}
\hline \multicolumn{1}{c}{ Source } & $M S_{\mathrm{e}}$ & $F$ & $p$ \\
\hline Latencies: Overall analysis & & & \\
Intensity & 15,086 & 90.56 & $<.001$ \\
Frequency & 3,170 & 91.88 & $<.001$ \\
Regularity & 2,144 & 31.35 & $<.001$ \\
Intensity $\times$ frequency & 2,773 & $<1$ & n.s. \\
Intensity $\times$ regularity & 1,410 & $<1$ & n.s. \\
Frequency $\times$ regularity & 1,207 & 15.64 & $<.001$ \\
Intensity $\times$ frequency $\times$ regularity & 1,613 & 1.57 & n.s. \\
Latencies: By-regularity analyses & & & \\
Regular words & & & \\
$\quad$ Intensity & 6,848 & 101.85 & $<.001$ \\
$\quad$ Frequency & 1,014 & 79.78 & $<.001$ \\
$\quad$ Intensity $\times$ frequency & 1,394 & 2.09 & n.s. \\
Irregular words & & & \\
$\quad$ Intensity & 9,647 & 69.33 & $<.001$ \\
$\quad$ Frequency & 3,363 & 68.15 & $<.001$ \\
$\quad$ Intensity $\times$ frequency & 2,992 & $<1$ & n.s. \\
Errors: Overall analysis & & & \\
Intensity & 42.15 & $<1$ & n.s. \\
Frequency & 56.92 & 84.41 & $<.001$ \\
Regularity & 62.15 & 48.29 & $<.001$ \\
Intensity $\times$ frequency & 38.81 & $<1$ & n.s. \\
Intensity $\times$ regularity & 44.74 & 1.79 & n.s. \\
Frequency $\times$ regularity & 69.84 & 46.55 & $<.001$ \\
Intensity $\times$ frequency $\times$ regularity & 37.31 & $<1$ & n.s. \\
\hline
\end{tabular}


Table 4

ANOVA Summaries for the Overall and the By-Regularity Analyses in Experiment 3

\begin{tabular}{lccc}
\multicolumn{4}{c}{ By-Reguiarity Analyses in Experiment 3 } \\
\hline \multicolumn{1}{c}{ Source } & $M S_{\mathrm{c}}$ & \multicolumn{1}{c}{$F$} & \multicolumn{1}{c}{$p$} \\
\hline Latencies: Overall analysis & & & \\
$\quad$ Case & 4,574 & 42.44 & $<.001$ \\
Frequency & 1,362 & 140.33 & $<.001$ \\
Regularity & 1,617 & 40.01 & $<.001$ \\
Case $\times$ frequency & 1,475 & 2.45 & n.s. \\
Case $\times$ regularity & 1,623 & 4.04 & $<.052$ \\
Frequency $\times$ regularity & 1,003 & $<1$ & n.s. \\
Case $\times$ frequency $\times$ regularity & 1,791 & 7.18 & $<.012$ \\
Latencies: By-regularity analyses & & & \\
Regular words & & & \\
$\quad$ Case & 1,362 & 47.47 & $<.001$ \\
Frequency & 1,297 & 63.85 & $<.001$ \\
$\quad$ Case $\times$ frequency & 1,489 & 10.12 & $<.004$ \\
Irregular words & & & \\
$\quad$ Case & 4,835 & 28.13 & $<.001$ \\
Frequency & 1,069 & 102.25 & $<.001$ \\
$\quad$ Case $\times$ frequency & 1,778 & $<1$ & n.s. \\
Errors: Overall analysis & & & \\
Case & 52.17 & 11.08 & $<.003$ \\
Frequency & 66.27 & 83.41 & $<.001$ \\
Regularity & 59.22 & 45.64 & $<.001$ \\
Case $\times$ frequency & 39.03 & 2.31 & n.s. \\
Case $\times$ regularity & 49.03 & $<1$ & n.s. \\
Frequency $\times$ regularity & 49.67 & 31.71 & $<.001$ \\
Case $\times$ frequency $\times$ regularity & 47.68 & 1.11 & n.s. \\
\hline & & & \\
& & &
\end{tabular}

regular than to irregular words. More importantly, and in contrast to Experiment 1, the three-way interaction between stimulus intensity, frequency, and regularity was not significant. This evidence for additivity shows that lowering stimulus intensity slows encoding but does not affect postencoding processes in the word recognition system.

Errors. Percent naming errors (see Table 1) were analyzed with a 2 (stimulus intensity: normal vs. low) $\times 2$ (frequency: high vs. low) $\times 2$ (regularity: regular vs. irregular) repeated measures ANOVA (see summary in Table 3). Fewer errors were made naming high-frequency $(2.2 \%)$ than low-frequency $(9.9 \%)$ words, and more errors were made naming irregular $(9.1 \%)$ than regular words $(3.0 \%)$. The frequency $\times$ regularity interaction was significant: For high-frequency words, there was no difference in errors to regular $(2.3 \%)$ versus irregular items $(2.1 \%)$. In contrast, for low-frequency words, substantially more errors were made to irregular $(16.2 \%)$ than to regular words $(3.7 \%)$. No other effects were significant.

Summary. The results show that stimulus intensity was additive with frequency and regularity. We conclude that whereas changes in stimulus intensity affect encoding, case alternation disrupts processing in the word recognition system at a postencoding stage. However, this conclusion is qualified somewhat by methodological differences between Experiments 1 and 2. Specifically, although the same stimulus words were used in both experiments, the presentation conditions differed. In Experiment 1 , lowercase and case-alternated stimuli were randomly intermixed within the stimulus sets. In Exper- iment 2, separate blocks were used for the normal- versus the low-illumination conditions. It is possible that the different patterns of results were due to differences in blocked versus randomized presentation of the stimuli.

\section{EXPERIMENT 3}

The purpose of Experiment 3 was to determine whether the pattern of results found in Experiment 1 would replicate when a blocking procedure similar to that of Experiment 2 was used. One block of trials contained words presented in lowercase and another block contained words in case-alternated format.

\section{Method}

Subjects. A total of 40 undergraduate students at Carleton University ( 20 male, 20 female) were recruited to participate; they received either course credit or $\$ 5$. All of the subjects had normal or corrected-to-normal vision and were native English speakers. None of the subjects had participated in the previous experiments.

Apparatus, Design, and Materials. The apparatus, design, and stimulus materials were identical to those used in Experiment 1 . As in Experiment 2, words were separated into two blocks of 40 words: One block of words was presented in lowercase format and the other in alternated-case format. Four orders were used, with each order consisting of two blocks. Each block contained 20 high- and 20 low-frequency words, with half of each being regular and the other half irregular.

Procedure. The procedure was identical to that of Experiment 2. The order of blocks (i.e., lowercase, alternated case) was counterbalanced across subjects. Subjects completed 10 practice trials before each of the 40 lowercase and 40 alternated-case trials.

\section{Results and Discussion}

Latencies. Latencies to correct responses are shown in Figure 2 (right panel) and Table 1. Latencies were examined with a 2 (case: lower vs. alternated) $\times 2$ (frequency: high vs. low) $\times 2$ (regularity: regular vs. irregular) repeated measures ANOVA. The ANOVA summary is shown in Table 4 . All three main effects were significant. Words were named faster in lowercase than in alternatedcase format, high-frequency words were named faster than low-frequency words, and regular words were named faster than irregular words.

Of primary interest was that the three-way interaction of case $\times$ frequency $\times$ regularity was significant. As shown in Figure 2 (right panel), the pattern of results replicated that of Experiment 1 very closely. For regular words, case alternation slowed naming more to low-than to high-frequency words. For irregular words, case alternation slowed naming of low- and high-frequency words equally. These patterns were confirmed with separate 2 (case: lower vs. alternated) $\times 2$ (frequency: high vs. low) analyses (see ANOVA summary in Table 4). For regular words, there were significant main effects of case and frequency. More importantly, the interaction between case and frequency was significant, thus replicating the pattern of results reported by Besner and McCann (1987) and that found in Experiment 1 of the present research. 
For irregular words, there were also main effects of case and frequency. However, as in Experiment 1 (see also Besner, 1990), these two factors did not interact.

Errors. Percent naming errors (see Table 1) were analyzed with a 2 (case: lower vs. alternated) $\times 2$ (frequency: high vs. low) $\times 2$ (regularity: regular vs. irregular) repeated measures ANOVA. The ANOVA summary is shown in Table 4. More errors were made on casealternated $(6.8 \%)$ than on lowercase $(4.7 \%)$ trials, lowfrequency $(10.2 \%)$ than on high-frequency $(1.4 \%)$ words, and irregular $(8.4 \%)$ than on regular $(3.1 \%)$ words. The frequency $\times$ regularity interaction in the latency data was supported in the error data. For high-frequency words, there was a negligible difference in naming errors to irregular $(2.1 \%)$ versus regular words $(0.7 \%)$. In contrast, for low-frequency words, substantially more errors were made naming irregular $(14.8 \%)$ than regular words $(5.6 \%)$.

Summary. In Experiment 3, lowercase and casealternated stimuli were presented in separate blocks. The results replicate those reported in Experiment 1, in which lowercase and case-alternated stimuli were randomly intermixed. This replication shows that, for regular words, case alternation and frequency interact. In contrast, for irregular words, case alternation and frequency are additive. As discussed in Experiment 1, the co-occurrence of these two patterns of results cannot be explained within the two-account/dual-route framework.

\section{GENERAL DISCUSSION}

The present research examined the relations among case alternation, word frequency, and word regularity. In Experiment 1, there was a significant three-way interaction among these factors (Figure 2, left panel). For regular words, case alternation slowed naming more to lowthan to high-frequency words. For irregular words, case alternation slowed naming of low- and high-frequency words equally. The results of Experiment 1 appear to be robust: The three-way interaction and the pattern of results associated with this interaction were closely replicated in Experiment 3 (Figure 2, right panel). Experiment 2 was designed to provide information concerning the locus of case alternation versus stimulus intensity effects in naming. Subjects named words presented either in normal or in low-illumination viewing conditions. In contrast to the interactions among case alternation and lexical factors found in Experiments 1 and 3, stimulus intensity was additive with frequency and regularity (Figure 2, middle panel): Whereas stimulus intensity affects encoding, case alternation affects processing at a postencoding stage in the word recognition system.

The complete pattern of present results cannot be addressed using either Account 1 or Account 2 of Besner and colleagues' two-account/dual-route approach. According to Account 1 (Besner \& Johnston, 1989), case alternation slows processing more along the nonlexical than the lexical route. On this view, for regular words, the interaction between case alternation and frequency oc- curred because naming of low-frequency regular words often relies on output not only from the lexical route but also from the nonlexical route. This account, however, does not address the finding that for irregular words, case alternation and frequency were additive: Case alternation slowed naming of high- and low-frequency irregular words equally. As discussed earlier, the assumption that case alternation slows the nonlexical route more than the lexical route incorrectly predicts that naming of low-frequency irregular words should have been faster in the case-alternated than in the lowercase format.

Account 2 hinges on the assumption that the presence of irregular words in the stimulus list orients processing toward the lexical route (Besner, 1990). This account subsumes Account 1: If processing has been oriented toward the lexical route, then the Account 1 assumption that case alternation slows processing more along the nonlexical than the lexical route is irrelevant. Account 2 can be used to address the present finding that, for irregular words, case alternation and frequency were additive. However, the assumption that processing was strategically oriented toward the lexical route leads to the erroneous prediction that case alternation and frequency should have been additive not only for irregular words but also for regular words. The present finding that, for regular words, case alternation and frequency interact, cannot be explained by appealing to the assumption that the presence of irregular words strategically oriented processing toward the lexical route.

In sum, the present research provided a critical test of the two-account/dual-route approach by examining the relation between case alternation and frequency under mixed-list conditions in which both regular and irregular words were presented. The two-account/dual-route approach did not pass this test. The complete pattern of results cannot be addressed either by assuming that case alternation slows processing more along the nonlexical than the lexical route (Besner \& Johnston, 1989) or by assuming that the presence of irregular words strategically orients processing toward the lexical route (Besner, 1990).

\section{Multilevels Approach}

Dual-route theorists unanimously agree that phonological coding involves processing along a lexical and a nonlexical route. However, there has been less agreement concerning the type of sublexical knowledge that is used by the nonlexical route. For example, Coltheart (1978) described nonlexical processing as the mapping of grapheme-to-phoneme correspondences (GPCs). Others (e.g., Kay \& Bishop, 1987; Patterson \& Morton, 1985) have suggested that nonlexical processing may also involve mapping orthographic bodies onto phonological rimes. The notion that processing along the nonlexical route involves various types of sublexical knowledge suggests that the dual-route system is really a multiroute, or multilevel, system. Such a system has been articulated by Shallice, Warrington, and McCarthy (1983; see also Shallice \& McCarthy, 1985). 
In this section, we outline a streamlined version of Shallice et al.'s (1983) multilevel system, which has been described and implemented by Norris (1994). The influence of case alternation on naming performance is then considered within the context of Norris's multilevel model, and a simulation of the present data showing interactions among case alternation, frequency, and regularity is generated.

Shallice et al. (1983; see also Shallice \& McCarthy, 1985) suggested that correspondences between orthography and phonology exist at a word level as well as at seven sublexical levels: initial consonant/consonant clusters, vowels, syllable final consonant cluster, initial consonant/consonant cluster plus vowel, rimes (bodies), syllables, and morphemes. These levels are assumed to be activated in parallel, with output from all levels being integrated into a unitary interpretation. Norris's (1994) version of this multilevel model was specifically tailored to deal with the corpus of 2,897 monosyllabic words used by Seidenberg and McClelland (1989). ${ }^{5}$ For monosyllabic words, the syllable, morpheme, and word units are functionally identical. Therefore, Norris represented these three units by a single word level, reducing the number of orthographic input levels from eight to six: initial consonant/consonant cluster, initial consonant/consonant cluster plus vowel, vowel, body, final consonant/consonant cluster, and word.

To implement this system, Norris (1994) added the multilevel representational scheme to a two-layer interactive-activation network that functions to combine outputs from the various levels. The implemented model maps the six levels of orthographic units at the input layer onto three sets of phonological units at the output layer. The three sets of output units correspond to initial consonant/ consonant cluster, vowel, and the final consonant/consonant cluster. Each unit within a set of output units represents an alternative pronunciation of the corresponding input. In order for an unambiguous pronunciation of a word to be generated, all units within a set are interconnected via bidirectional inhibitory links. The three sets of output nodes are not connected. The orthographic input and phonologic output units are connected with feedforward facilitatory links that encode the correspondences that have been acquired through exposure to a corpus of words. These correspondences are coded as an initial set of weightings.

In establishing parameter values for his implemented model, Norris (1994) originally allowed each of the six input-output levels to have different weightings. Simulations showed, however, that only three different weights were necessary in order for the model to operate effectively. These three weightings reflect the size of the input units: phoneme units (initial consonant/consonant cluster, vowels, final consonant/consonant cluster), CV and body units, and word units. ${ }^{6}$ The higher the weighting, the more influential the correspondences are at that level.

In dual-route models, regularity effects are attributed to overlap between codes generated from the two routes:
Irregular words are processed slowly because the lexical (correct) code receives competition from the nonlexical (incorrect) code in the response buffer. In the multilevel model, inputs from all of the levels are considered in parallel. Any conflict that exists within a level is resolved through inhibition. In contrast to dual-route models, this means that regularity effects also occur at sublexical levels in the multilevel model. For example, presenting the word head will activate the orthographic units representing $h, e a, d$, hea, ead, and head. Because the grapheme $e a$ can correspond to either $/ \varepsilon /$ or $/ \mathrm{i} /$, the input units activate the phoneme units for both $/ \varepsilon /$ and $/ \mathrm{i} /$ in the output layer. The phoneme nodes $/ \varepsilon /$ and $/ \mathbf{i} /$ are connected by inhibitory links. As the network cycles, the competition between alternative pronunciations (e.g., / $/ \varepsilon /$ and $/ \mathrm{i} /$ ) is slowly resolved and the network settles on a single, unambiguous pronunciation. The settling process will take longer if the activations of the alternative pronunciations are evenly balanced.

The sublexical correspondences from the orthographic units to the phoneme units are coded as a function of type frequency (i.e., a count of all the different words in the corpus encountered by the model) of which a particular correspondence occurs. In processing a particular word through the model, type frequency is multiplied by the weight parameter for that level. By using type frequency, the sublexical correspondences will have higher type counts for regular words (e.g., mint, tint, flint, hint) than for competing irregular words (e.g., pint).

Pronunciation latency is a function of the number of cycles the network takes to reach a response criterion. There are two components to the response criterion. First, activation in the network (specifically, activation of the phoneme units corresponding to the vowel) must exceed a particular threshold. However, in addition to exceeding a threshold, the activation for the most active phoneme must exceed other activations by a given margin. So, if one phoneme passes the threshold but another has a very similar level of activation, the network will continue to cycle until the most active phoneme eventually wins by a given margin.

In the multilevel model, word frequency influences processing at the word level. This is achieved by multiplying the weighting at the word level by the log word frequency (Kučera \& Francis, 1967) for the item being processed. Accordingly, for high-frequency words, wordlevel information (from the head $\rightarrow /$ hed/ correspondence) will generally dominate the activation process, thereby producing a fast naming response that is minimally influenced by sublexical activation and thus is insensitive to regularity. For low-frequency words, activation at the word level will be weaker, and sublexical information will play a role. If the low-frequency word is irregular, then competition at the sublexical level will take longer to be resolved, thereby resulting in longer naming latencies than if the word were regular.

Simulating case alternation. Our goal was to use Norris's (1994) implemented model to simulate the effects of 
Table 5

Settings for the Nine Free Parameters Used in the Multilevel Simulation

\begin{tabular}{lcc}
\hline & \multicolumn{2}{c}{ Condition } \\
\cline { 2 - 3 } Free Parameter & Lowercase & Case Alternation \\
\hline Phoneme weight & 1.055 & 1.055 \\
CV/body weight & 1.736 & 1.736 \\
Word weight & 15.701 & 11.000 \\
Inhibition & 1.455 & 1.455 \\
Threshold & 0.817 & 0.817 \\
Margin & 0.352 & 0.352 \\
Scalar & 18.472 & 18.472 \\
RT offset & 560.977 & 588.000 \\
Extra frequency & 0.186 & 0.186 \\
\hline
\end{tabular}

Note-Parameter values were obtained by fitting the model to the lowercase data and then by adjusting only the word-level weight and the RT-offset value to accommodate the effect of case alternation. RT, reaction time.

case alternation on word processing. To this end, our first step was to "construct" the model by establishing the correspondences for the phoneme level, the $\mathrm{CV} /$ body level, and the word level. As in Norris, correspondences were acquired by exposing the model to the entire set of 2,897 monosyllabic words in the Seidenberg and McClelland (1989) corpus: As noted, the model learns and codes the sublexical correspondences according to type frequency and the word-level correspondences according to word frequency. Next, the parameter values were set by fitting the model to the lowercase data obtained in the present experiments. For this, we averaged the data from Experiments 1 and 3 of the present research (see Figure 3, darker lines). As shown in Table 5, the model fitting includes nine free parameters, of which three weightings are for the different levels of input-output correspondence (i.e., phoneme level, CV/body level, word level). Fitting was accomplished using a version of a simplex algorithm (Nelder \& Mead, 1965). The simulated data are shown in Figure 3 (lighter lines). As can be seen in Figure 3, the simulated data closely match the frequency, regularity, and frequency $\times$ regularity effects found in the lowercase condition of the present research.

Having established the correspondences for the model and having set the parameters by fitting the model to the data from the lowercase condition, we next simulated the disruptive effects of case alternation. In the multilevel model, the disruptive effects of case alternation are interpreted as (1) the addition of a constant to the reaction time (RT)-offset value to reflect the extra time required to fully encode and parse words in the case-alternation condition as opposed to the lowercase condition, and (2) the lowering of weightings for one or more of the three input-output correspondences. In deciding which of the input-output weights to lower, we assumed that case alternation disrupts processing only at the word level. This assumption was based on the notion that, in the multilevel model, activation at the word level is influenced by the processing of holistic units: Case alternation affects this processing by disrupting the integrity of the interletter patterns in the holistic units. ${ }^{7}$ Therefore, we kept all of the sublexical parameters unchanged and simply lowered the weight at the word level. The nine parameter values for the lowercase and for the casealternated simulations are shown in Table 5. As shown in Figure 3 (compare darker vs. lighter lines), by adding $27 \mathrm{msec}$ to the RT offset (from 561 to 588) for a general slowing and by lowering only the word-level weight (from 15.70 to 11.00 ), we were able to simulate the effects of case alternation.

Why is it that the case alternation $\times$ frequency $\times$ regularity interaction, which provides such a challenge to the dual-route framework, can be simulated using the multilevel model by simply increasing RT offset and lowering the weighting of the word level? As discussed below, the answer lies in the locus of regularity effects. Whereas the dual-route model codes regularity as an interaction between lexical and sublexical routes, the multilevel model codes regularity at a sublexical level.

High-frequency words. For high-frequency regular words, the effect of case alternation in the human data is completely accounted for in the simulation with the increase to the RT-offset parameter. One might wonder why lowering the word-level weight did not impose an additional change in time to process high-frequency regular items, given that the word level plays a role in generating an output phonology for high-frequency words. Because the sublexical correspondences are consistent for high-frequency regular words, the combination of the sublexical contribution with the diminished word-level contribution remains sufficient to maintain performance at close to a ceiling level. The fact that ceiling or nearceiling level of performance has been achieved is evident

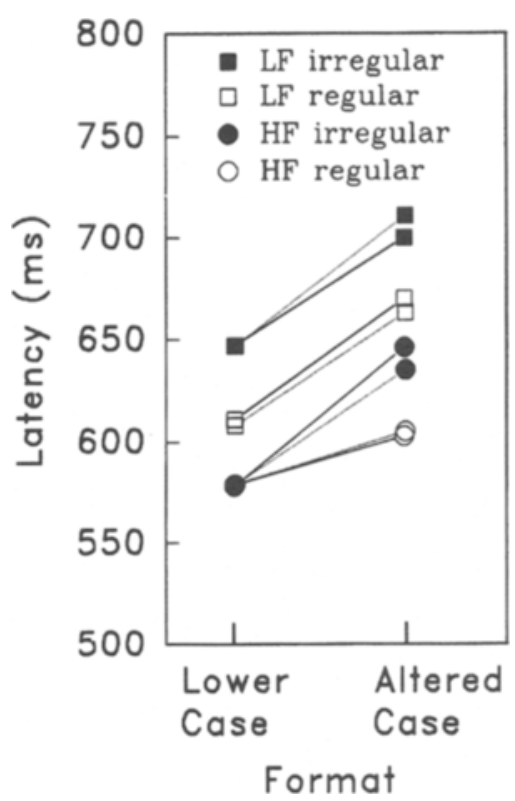

Figure 3. Simulated data (lighter lines) from the multilevel model and human data averaged across Experiments 1 and 3 (darker lines). LF, low frequency; HF, high frequency. 
in the fact that the model processes high-frequency regular words in just one cycle: The 606-msec naming time (case-alternation condition) estimated by the model represents 1 cycle $* 18.5 \mathrm{msec}$ SCALER +588 -msec offset. To demonstrate that the word level is still playing an important role in naming high-frequency regular words in the case-alternation condition, we ran a simulation in which the RT offset for the case-alternation condition was used, but the word-level weight was set to zero. Under these conditions, the model overestimated naming of high-frequency regular words by $117 \mathrm{msec}$. So, although the model's sublexical mappings can generate responses to high-frequency regular items, a contribution from the word-level still facilitates this process in the casealternation simulation even though we lowered the wordlevel weight from 15.701 to 11.000 .

For high-frequency irregular words, naming under normal presentation conditions (i.e., lowercase) is based predominantly on input from the word level: The fact that the sublexical level does not play a significant role is reflected in the finding that high-frequency words do not show regularity effects in either human data or our simulation. The lowering of the word-level weight in our simulation of case alternation is sufficient to make the output sensitive to the inconsistent mappings that are activated at the sublexical level in the face of a highfrequency irregular word. That is, although the system is close to ceiling for high-frequency words, lowering the word-level weighting allows the disruptive sublexical coding of irregularity to affect the latencies of high-frequency irregular words. This is reflected in the extra slowing beyond the 27-msec change in the RT offset.

Low-frequency words. Under normal presentation conditions (e.g., lowercase), naming is sensitive to frequency because low-frequency words do not receive as much benefit as high-frequency words from word-level input. Accordingly, sublexical coding plays a stronger role for low- than for high-frequency words in the activation of phonology at the output level. As noted, because type frequency is used to code regularity at the sublexical levels, more cycles are required to resolve lowfrequency irregular words as opposed to low-frequency regular words, thereby accounting for the regularity effect in naming of low-frequency words. Our human data show that case alternation slowed naming of low-frequency regular and irregular words equally by an average of $56 \mathrm{msec}$. This was closely replicated in our simulation with an average slowing of $61 \mathrm{msec}$ for low-frequency words. As with high-frequency words, $27 \mathrm{msec}$ of the effect is simulated as general slowing by increasing the RToffset parameter by $27 \mathrm{msec}$. The remaining effect of case alternation is attributable to the lowering of the wordlevel weight. Our simulation, therefore, shows that although low-frequency words benefit less than highfrequency words from word-level input, under normal presentation conditions (i.e., lowercase), the word-level still contributes to the phonological output of lowfrequency words. To further demonstrate this point, we ran the low-frequency items through a simulation in which the word-level weight was set to zero. Under these conditions, the model was unable to name low-frequency irregular words accurately (only one low-frequency irregular item was named correctly), showing that phonological coding of low-frequency irregular words requires input from the word level. Moreover, when the word-level contribution was zeroed, latencies were the same to lowfrequency regular as to high-frequency regular items (726 and $729 \mathrm{msec}$, respectively). As expected, when the wordlevel contribution is removed, the sublexical contributions to regular words are the same regardless of frequency.

\section{Conclusions}

The importance of the present research is in showing that variations in presentation format influence processing within the lexical system, and in particular, that presenting words in lowercase versus case-alternated format interacts with word frequency and regularity. Using an implemented version of Norris's (1994) multilevel model, we simulated the interactions between presentation format and lexical factors by (1) adding a constant to the RToffset parameter to reflect extra time to fully encode and parse words in the case-alternated condition and (2) by lowering the word-level contribution to phonological output. In contrast, the two-account/dual-route framework cannot explain the fact that when regular and irregular items are presented together in a mixed list, case alternation and frequency have additive effects on naming of irregular words but interact for naming of regular words.

We have considered only the effects of case alternation within the context of dual-route and multilevel models for the simple reason that these models provide ways through which case alternation can influence processing beyond an initial encoding stage. In the dual-route model, case alternation disrupts letter and multiletter processing. In the multilevel model, case alternation is assumed to disrupt encoding/parsing of words and the weighting connecting whole-word orthography to phonological units. Parallel-distributed-processing (PDP) models (Plaut, McClelland, Seidenberg, \& Patterson, 1996; Seidenberg \& McClelland, 1989) do not provide mechanisms that would allow for presentation format to interact with lexical (network) factors. In PDP models, access into the lexical network is modeled as a layer of orthographic input units. The orthographic-input layer provides an abstract representation of letter strings in such a way that presenting letter strings in lowercase versus case-alternated format cannot affect processing in the input layer. Therefore, in PDP systems, the disruptive influence of case alternation must occur prior to the network and thus cannot interact with frequency and regularity, which are considered to influence processing within the lexical network. The present findings, therefore, present a challenge to 
PDP models and to any other model that implements an abstract representational scheme at the input layer.

\section{REFERENCES}

Adams, M. J. (1979). Models of word recognition. Cognitive Psychology, 11, 133-176.

BALUCH, B., \& BESNER, D. (1991). Visual word recognition: Evidence for strategic control of lexical and nonlexical routines in oral reading. Journal of Experimental Psychology: Learning, Memory, \& Cognition, 17, 644-652.

BerNSTEIN, S. E., \& CARR, T. H. (1996). Dual route theories of spelling to pronunciation: What can be learned from concurrent task performance? Journal of Experimental Psychology: Learning, Memory, \& Cognition, 22, 86-116.

BESNER, D. (1990). Does the reading system need a lexicon? In D. A. Balota, G. B. Flores d'Arcais, \& K. Rayner (Eds.), Comprehension processes in reading (pp. 73-99). Hillsdale, NJ: Erlbaum.

BESNER, D., \& JOHNSTON, J. C. (1989). Reading and the mental lexicon: On the uptake of visual information. In W. Marslen-Wilson (Ed.), Lexical representation and process (pp. 291-316). Cambridge: Cambridge University Press.

BESNER, D., \& MCCANN, R. S. (1987). Word frequency and pattern distortion in visual word identification and production: An examination of four classes of models. In M. Coltheart (Ed.), Attention and performance XII: The psychology of reading (pp. 201-219). Hillsdale, NJ: Erlbaum.

BESNER, D., \& SMITH, M. C. (1992). Models of visual word recognition When obscuring the stimulus yields a clearer view. Journal of Experimental Psychology: Learning, Memory, \& Cognition, 18, 468-482.

BESNER, D., \& SWAN, M. (1982). Models of visual word recognition. Quarterly Journal of Experimental Psychology, 34A, 313-325.

CARR, T. H., \& Pollatsek, A. (1985). Recognizing printed words: A look at current models. In D. Besner, T. G. Waller, \& G. E. MacKinnon (Eds.), Reading research: Advances in theory and practice (Vol. 5 , pp. 1-82). Orlando, FL: Academic Press.

ColthearT, M. (1978). Lexical access in simple reading tasks. In G. Underwood (Ed.), Strategies of information processing (pp. 151-216). San Diego: Academic Press.

Coltheart, M., Curtis, B., Atkins, P., \& Haller, M. (1993). Models of reading aloud: Dual-route and parallel-distributed-processing approaches. Psychological Review, 100, 589-608.

Coltheart, M., \& Freeman, R. (1974). Case alternation impairs word identification. Bulletin of the Psychonomic Society, 3, 102-104.

Herdman, C. M., \& BecKetT, B. L. (1996). Code-specific processes in word naming: Evidence supporting a dual-route model of word recognition. Journal of Experimental Psychology: Human Perception \& Performance, 22, 1149-1165.

Herdman, C. M., LeFevre, J., \& Greenham, S. L. (1994). Implicating the lexicon: Base-word frequency effects in pseudohomophone naming. Journal of Experimental Psychology: Human Perception \& Performance, 20, 575-590.

KAY, J., \& BISHOP, D. (1987). Anatomical differences between Nose, Palm, and Foot, or, the body in question: Further discussion of the processes of sub-lexical spelling-sound translation. In M. Coltheart (Ed.), Attention and performance XII: The psychology of reading (pp. 449-469). Hillsdale, NJ: Erlbaum.

KUČERA, H., \& FRANCIS, W. N. (1967). Computational analysis of presentday American English. Providence, RI: Brown University Press.

MAsson, M. E. J. (1986). Identification of typographically transformed words: Instance-based skill acquisition. Journal of Experimental Psychology: Learning, Memory, \& Cognition, 12, 479-488.

MCCANN, R. S., \& BESNER, D. (1987). Reading pseudohomophones: Implications for models of pronunciation assembly and the locus of word-frequency effects in naming. Journal of Experimental Psychology: Human Perception \& Performance, 13, 14-24.

Monsell, S., Patterson, K. E., Graham, A., Hughes, C. H., \& MilROY, R. (1992). Lexical and sublexical translation of spelling to sound:
Strategic anticipation of lexical status. Journal of Experimental Psychology: Learning, Memory, \& Cognition, 18, 452-467.

MORTON, J. (1969). Interaction of information in word recognition. Psychological Review, 76, 165-178.

Nelder, J. A., \& MEAD, R. (1965). A simplex method for function minimization. Computer Journal, 7, 308-313.

NORRIS, D. (1994). A quantitative multiple-levels model of reading aloud. Journal of Experimental Psychology: Human Perception \& Performance, 20, 1212-1232.

PaAp, K. R., McDonald, J. E., Schvaneveldt, R. W., \& Noel, R. W. (1987). Frequency and pronounceability in visually presented naming and lexical decision tasks. In M. Coltheart (Ed.), Attention and performance XII: The psychology of reading (pp. 221-243). Hillsdale, NJ: Erlbaum.

PAAP, K. R., \& NOEL, R. W. (1991). Dual-route models of print to sound: Still a good horse race. Psychological Research, 53, 13-24.

Patterson, K. E., \& Morton, J. (1985). From orthography to phonology: A new attempt at an old interpretation. In K. E. Patterson, J. C. Marshall, \& M. Coltheart (Eds.), Surface dyslexia: Neuropsychological and cognitive studies of phonological reading (pp. 335-359). Hillsdale, NJ: Erlbaum.

Peereman, R., \& Content, A. (1995). Neighborhood size effect in naming: Lexical activation or sublexical correspondences? Journal of Experimental Psychology: Learning, Memory, \& Cognition, 21, 409-421.

Plaut, D. C., McClelland, J. L., Seidenberg, M. S., \& Patterson, K. (1996). Psychological Review, 103, 56-115.

Pugh, K. R., Rexer, K., \& KATZ, L. (1994). Evidence of flexible coding in word recognition. Journal of Experimental Psychology: Human Perception \& Performance, 20, 807-825.

Seidenberg, M. S., \& MCClelland, J. L. (1989). A distributed, developmental mental model of word recognition and naming. Psychological Review, 96, 523-568.

Seidenberg, M. S., Waters, G. S., Barnes, M. A., \& Tannenhaus, M. K. (1984). When does irregular spelling or pronunciation influence word recognition? Journal of Verbal Learning \& Verbal Behavior, 23, 383-404.

Shallice, T., \& MicCarthy, R. (1985). Phonological reading: From patterns of impairment to possible procedures. In K. E. Patterson, J. C. Marshall, \& M. Coltheart (Eds.), Surface dyslexia: Neuropsychological and cognitive studies of phonological reading (pp. 361-397). Hillsdale, NJ: Erlbaum.

Shallice, T., Warrington, E. K., \& McCarthy, R. (1983). Reading without semantics. Quarterly Journal of Experimental Psychology, 35A, 111-138.

Stanners, R. F., Jastrzembski, J. E., \& Westbrook, A. (1975). Frequency and visual quality in a word-nonword classification task. Journal of Verbal Learning \& Verbal Behavior, 14, 259-264.

STERnBERG, S. (1969). The discovery of processing stages: Extension of Donder's method. In W. G. Koster (Ed.), Attention and performance II. Amsterdam: North-Holland

WATERS, G. S., \& SEIDENBERG, M. S. (1985). Spelling-sound effects in reading: Time-course and decision criteria. Memory \& Cognition, 13 , $557-572$.

\section{NOTES}

1. The present research was focused on the effects of case alternation in naming. An extended dual-route architecture has been outlined by Besner (1990; Besner \& Johnston, 1989) to address case-alternation effects in lexical decisions.

2. Another explanation of this interaction is that case alternation affects the rate at which frequency-sensitive processes are activated in the orthographic lexicon (Besner \& Johnston, 1989). This explanation hinges on the assumption that letter identification feeds information into the orthographic lexicon in a continuous (nondiscrete) manner such that slowing letter identification by presenting words in case-alternated format decreases the rate at which lexical units are activated in the orthographic lexicon. Besner (1990) had to dismiss this "rate-based" 
explanation, however, to formulate Account 2. For Account 2 to work, it must be assumed that letter identification is discrete from frequencysensitive processes in the orthographic lexicon.

3. Luminance was measured by placing the aperture of a Tektronics J16 photometer inside a $1 \times 1$ in. square of light that was centered at the location where words were presented on the video screen.

4. A 2 (case: lower vs. alternated) $\times 2$ (regularity: regular vs. irregular) repeated measures ANOVA of the high-frequency words showed a significant interaction $\left[F(1,39)=14.85, M S_{\mathrm{e}}=1,331.68, p<.001\right]$, where high-frequency regular words were less affected by case alterna- tion than were high-frequency irregular words. For low-frequency words, the interaction between case and regularity was not significance $(F<1)$.

5 . All of the words in the present research were monosyllabic.

6. Using weightings for each of the six levels would generate a slightly more precise model but would increase computational complexity.

7. This is a simplifying assumption that restricts our multilevel simulation by limiting the number of parameters that are allowed to vary. Case alternation may also disrupt processing at sublexical levels: Allowing the sublexical weights to vary would generate a more precise fit of the data in the case-alternation condition.

\section{APPENDIX}

Stimuli Used in Experiments 1-3

\begin{tabular}{lllll}
\hline \multicolumn{2}{c}{ High-Frequency } & & \multicolumn{2}{c}{ Low-Frequency } \\
\cline { 5 - 5 } Regular & Irregular & & Regular & Irregular \\
\hline group & put & & wig & pear \\
must & great & & chop & sweat \\
week & shall & & carve & flood \\
club & both & & dusk & mow \\
page & said & & snag & aunt \\
dark & come & clam & worm \\
which & are & float & soot \\
hope & want & rang & caste \\
block & were & plank & spook \\
more & warm & peel & bush \\
best & done & wink & wand \\
place & does & pump & sew \\
soon & gross & flung & swamp \\
did & work & beam & wool \\
stop & do & deed & warp \\
neck & move & sank & wad \\
made & says & gum & pint \\
can & break & frog & deaf \\
we & watch & grape & plaid \\
while & give & nut & bowl \\
\hline & & &
\end{tabular}

(Manuscript received June 21, 1995;

revision accepted for publication December 9, 1997.) 